\title{
Spontaneous Emission from Photonic Crystals: Full Vectorial Calculations
}

\author{
Zhi-Yuan Li, ${ }^{1,2}$ Lan-Lan Lin, ${ }^{2}$ and Zhao-Qing Zhang ${ }^{1}$ \\ ${ }^{1}$ Department of Physics, Hong Kong University of Science and Technology, Clear Water Bay, Kowloon, Hong Kong, China \\ ${ }^{2}$ Institute of Physics, Chinese Academy of Sciences, P.O. Box 603, Beijing 100080, China
}

(Received 11 October 1999)

\begin{abstract}
Quantum electrodynamics of atom spontaneous emission from a three-dimensional photonic crystal is studied in a full vectorial framework. The electromagnetic fields are quantized via solving the eigenproblem of photonic crystals with use of a plane-wave expansion method. It is found that the photon density of states and local density of states (LDOS) with a full band gap vary slowly near the edge of band gap, in significant contrast to the singular character predicted by the previous isotropic model. Therefore, the spontaneous emission can be solved by conventional Weisskopf-Wigner approximate theory, which yields a pure exponentially decaying behavior with a rate proportional to the LDOS.
\end{abstract}

PACS numbers: 42.70.Qs, 32.80.-t, 42.50.Dv

In recent years, there has been vast interest in fabrication of photonic crystals with a full band gap [1-3]. In the band gap, there is no electromagnetic (EM) mode that can propagate in the crystal, which results in some peculiar physical properties, such as inhibition of atom spontaneous emission and localization of light [2,3]. Recently, much progress has been made toward fabricating threedimensional (3D) photonic crystals with an absolute band gap in visible and infrared regimes by means of microlithography and inverse-opal technique [4-6].

Another important subject that has attracted much attention concerns the understanding of quantum electrodynamics (QED) behaviors of atoms and molecules in photonic crystals. In the theoretical side, an isotropic model developed by John and co-workers [7] was almost exclusively adopted to treat the QED problems by this group and other groups. In that model, the dispersion relation of photon in one-dimensional periodic multilayers is extended directly to the 3D case. This results in a singular behavior of photon density of states (DOS) as proportional to $\left(\omega-\omega_{c}\right)^{-1 / 2}$, where $\omega_{c}$ is the edge frequency of the band gap. As the photon DOS plays an important role in determining the QED behavior of atoms when their emission frequency lies near the gap edge, some peculiar properties were predicted by this model, such as the anomalous Lamb shift [7], oscillatory behavior of spontaneous emission [8], and enhanced quantum interference effects [9]. In addition, the vectorial nature of EM fields is completely omitted. Although an improved model employing more realistic photon dispersion as $\omega=\omega_{c}+A\left(\mathbf{k}-\mathbf{k}^{\prime}\right)^{2}$ was discussed in these works $[7,8,10]$, the extension of the dispersion formula near the gap edge to the whole momentum space and the neglect of vectorial nature of EM fields remain.

On the other hand, the spontaneous emission and fluorescence of molecules in photonic crystals have been investigated by several groups [11-13]. However, there still is a lack of clear quantitative understanding of such QED characters observed in these experiments, as other effects such as complex chemical and electronic interactions may account for a major fraction of the change in the measured QED characters.

All these QED subjects require more accurate solution of light and atom interaction in the framework of quantum theory, fully taking into account the vectorial character of EM fields. In this Letter, we will investigate the QED of spontaneous emission in a 3D photonic crystal based on a full vectorial treatment of EM fields. It should be noted that the vectorial nature of EM waves is well established in studying photonic band structures [14,15], photon DOS [16], and dipole radiation [17] in 3D photonic crystals; however, the discussion of QED subject remains lying in the scalar framework [7-10].

The full quantum theory of light and atom interaction first requires the quantization of EM fields. In a 3D photonic crystal, we apply a plane-wave expansion method [14-16] to solve the eigenmodes. We denote $|n, \mathbf{k}\rangle$ as the Bloch state at the wave vector of $\mathbf{k}$ and the $n$th photonic band, which has a frequency $\omega_{n \mathbf{k}}$ and a magnetic field function of $\mathbf{H}_{n \mathbf{k}}(\mathbf{r})$. The eigenmodes satisfy the following orthogonalization and normalization relations: $\int_{V} \mathbf{H}_{n^{\prime} \mathbf{k}^{\prime}}^{*}(\mathbf{r}) \cdot \mathbf{H}_{n \mathbf{k}}(\mathbf{r}) d^{3} \mathbf{r}=V \delta_{n, n^{\prime}} \delta\left(\mathbf{k}-\mathbf{k}^{\prime}\right)$, where $V$ is the volume of photonic crystal. The eigenmode $\mathbf{H}_{n \mathbf{k}}$ obtained in this way is a nondimensional function.

Following the common way of photon quantization, we can expand the EM fields in the photonic crystal as follows:

$$
\begin{aligned}
\mathbf{H}(\mathbf{r}, t)=\sum_{n \mathbf{k}} h_{n \mathbf{k}}[ & \mathbf{H}_{n \mathbf{k}}(\mathbf{r}) a_{n \mathbf{k}} e^{-i \omega_{n \mathbf{k}} t} \\
& \left.+\mathbf{H}_{n \mathbf{k}}^{*}(\mathbf{r}) a_{n \mathbf{k}}^{\dagger} e^{i \omega_{n \mathbf{k}} t}\right],
\end{aligned}
$$

where $a_{n \mathbf{k}}$ and $a_{n \mathbf{k}}^{\dagger}$ are annihilation and creation operators of photon with a Bloch state $|n \mathbf{k}\rangle$. The coefficients can be derived as $h_{n \mathbf{k}}=\sqrt{\hbar \omega_{n \mathbf{k}} / 2 \mu_{0} V}$, so that the Hamiltonian of photon in a photonic crystal has the well-known standard form $H_{f}=\sum_{n \mathbf{k}} \hbar \omega_{n \mathbf{k}}\left(a_{n \mathbf{k}}^{\dagger} a_{n \mathbf{k}}+\frac{1}{2}\right)$.

The quantized electric field can be obtained by using Maxwell's equations: 


$$
\begin{aligned}
\mathbf{E}(\mathbf{r}, t)=\sum_{n \mathbf{k}}[ & \mathbf{E}_{n \mathbf{k}}(\mathbf{r}) a_{n \mathbf{k}} e^{-i \omega_{n \mathbf{k}} t} \\
& \left.+\mathbf{E}_{n \mathbf{k}}^{*}(\mathbf{r}) a_{n \mathbf{k}}^{+} e^{i \omega_{n \mathbf{k}} t}\right],
\end{aligned}
$$

with $\mathbf{E}_{n \mathbf{k}}(\mathbf{r})=\frac{i c}{\epsilon(\mathbf{r})} \sqrt{\hbar / 2 \epsilon_{0} \omega_{n \mathbf{k}} V} \nabla \times \mathbf{H}_{n \mathbf{k}}(\mathbf{r})$.

With the quantized EM fields, we can now solve the atomic QED via a standard full quantum theory. Consider a two-level atom with excited state $|2\rangle$, ground state $|1\rangle$, and resonant transition frequency $\omega_{0}$. The Schrödinger equation of the system in the interaction picture reads

$$
i \hbar \frac{\partial}{\partial t}|\psi(t)\rangle=H_{I}|\psi(t)\rangle
$$

where the interaction Hamiltonian is given as $H_{I}=$ $\sum_{n \mathbf{k}} \hbar\left(g_{n \mathbf{k}} \sigma_{+} a_{n \mathbf{k}} e^{i \Delta_{n \mathbf{k}} t}+g_{n \mathbf{k}}^{*} \sigma_{-} a_{n \mathbf{k}}^{+} e^{-i \Delta_{n \mathbf{k}} t}\right) . \quad$ Here $\Delta_{n \mathbf{k}}=\omega_{0}-\omega_{n \mathbf{k}}$, while $\sigma_{+}=|2\rangle\langle 1|$ and $\sigma_{-}=|1\rangle\langle 2|$ are atomic transition operators. The coupling coefficient of atom-field interaction in the dipole approximation reads $g_{n \mathbf{k}}=-\mathbf{u} \cdot \mathbf{E}_{n \mathbf{k}}(\mathbf{r}) / \hbar$, with $\mathbf{u}$ being the dipole moment vector of atomic transition between states $|2\rangle$ and $|1\rangle$.

Assume the atom is initially on the excited state $|2\rangle$ and the field is in the vacuum state. The wave function of the system then can be written as

$$
|\psi(t)\rangle=c_{2}(t)|2,\{0\}\rangle+\sum_{n \mathbf{k}} c_{1, n \mathbf{k}}(t)|1, n \mathbf{k}\rangle .
$$

Substituting the wave function into Schrödinger equation (3), we can derive the following formal differential-integral equation satisfied with $c_{2}(t)$ :

$$
\frac{d}{d t} c_{2}=-\sum_{n \mathbf{k}}\left|g_{n \mathbf{k}}\right|^{2} \int_{0}^{t} e^{i \Delta_{n \mathbf{k}}\left(t-t^{\prime}\right)} c_{2}\left(t^{\prime}\right) d t^{\prime}
$$

To solve this complex equation for spontaneous emission, some approximations should be made. For atoms in vacuum, the Weisskopf-Wigner theory is an excellent approximation, which results in a pure exponential-decay behavior of excited state. As is noted above, there remain two great simplifications in the isotropic model used for QED in photonic crystals. First the dispersion relation $\omega=\omega_{c}+A\left(k-k_{0}\right)^{2}$ is used for 3D crystals, and then it is extended to the whole momentum space, with the assumption that main contributions should come from the photon modes near the atomic resonant frequency. Another approximation neglects the dependence of atom-field coupling coefficient $g_{n \mathbf{k}}$, on the Bloch states. With these approximations, Eq. (5) can be analytically solved through a Laplace-transform method. However, the results are based on scalar wave approximation, and are quite doubtful.

To solve Eq. (5), we define the photon DOS $\rho(\omega)$ and the local density of states (LDOS) $\rho(\omega, \mathbf{r})$ for the photonic crystal as

$$
\begin{aligned}
\rho(\omega) & =\sum_{n \mathbf{k}} \delta\left(\omega-\omega_{n \mathbf{k}}\right) \\
& =\frac{V}{(2 \pi)^{3}} \sum_{n} \int_{\mathrm{BZ}} d^{3} \mathbf{k} \delta\left(\omega-\omega_{n \mathbf{k}}\right),
\end{aligned}
$$

$$
\begin{aligned}
\rho(\omega, \mathbf{r})= & \sum_{n \mathbf{k}}\left|g_{n \mathbf{k}}(\mathbf{r})\right|^{2} \delta\left(\omega-\omega_{n \mathbf{k}}\right) \\
= & \frac{u_{0}^{2} c^{2}}{2 \hbar \epsilon_{0}(2 \pi)^{3} \epsilon^{2}(\mathbf{r})} \\
& \times \sum_{n} \int_{\mathrm{BZ}} d^{3} \mathbf{k} \frac{\left|\hat{\mathbf{u}} \cdot\left[\nabla \times \mathbf{H}_{n \mathbf{k}}(\mathbf{r})\right]\right|^{2}}{\omega_{n \mathbf{k}}} \\
& \times \delta\left(\omega-\omega_{n \mathbf{k}}\right) .
\end{aligned}
$$

In Eqs. (6) and (7), BZ means the first Brillouin zone of photonic crystals, $u_{0}$ and $\hat{\mathbf{u}}$ denote the magnitude and direction of dipole moment $\mathbf{u}$, respectively. When the atomic dipole is randomly oriented in space, Eq. (7) can be further simplified.

The DOS and LDOS for a vacuum can be easily derived and take a simple form: $\rho(\omega)=4 V \omega^{2} /\left(2 \pi^{2}\right) c^{3}$ and $\rho(\omega, \mathbf{r})=\left(1 / 4 \pi \epsilon_{0}\right)\left(4 \omega^{3} u_{0}^{2}\right) /\left(6 \pi \hbar c^{3}\right)$. The LDOS does not depend on the position of atom in space. However, in a 3D photonic crystal, the analytical expression for the DOS and LDOS is not available. Instead, numerical calculation is necessary, which is complex and tedious. The full vectorial treatment involves several numerical steps: Discretize the Brillouin zone into many sampling cells, then solve the photonic bands and EM fields at each cell by the plane-wave expansion method, and finally calculate the DOS and LDOS by summing the contribution from all Bloch states via Eqs. (6) and (7). To improve the calculation accuracy, a modified tetrahedral integration method developed in electronic systems [18] is utilized.

With the definition of the DOS and LDOS, Eq. (5) now takes the form

$$
\frac{d}{d t} c_{2}=-\int_{0}^{\infty} \rho(\omega, \mathbf{r}) d \omega \int_{0}^{t} e^{i\left(\omega_{0}-\omega\right)\left(t-t^{\prime}\right)} c_{2}\left(t^{\prime}\right) d t^{\prime} .
$$

For the vacuum mode, the solution of Eq. (8) can be readily obtained by the Weisskopf-Wigner theory, and we have

$$
\frac{d}{d t} c_{2}=-\frac{\Gamma}{2} c_{2}, \quad \Gamma=2 \pi \rho\left(\omega_{0}, \mathbf{r}\right)=\frac{1}{4 \pi \epsilon_{0}} \frac{4 \omega_{0}^{3} u_{0}^{2}}{3 \hbar c^{3}} .
$$

Therefore, the atomic excited state decays in a purely exponential behavior, with $\tau=1 / \Gamma$ being its lifetime. The Weisskopf-Wigner theory is applicable if the DOS (more accurately the LDOS) of photon modes varies slowly near atomic resonant frequency $\omega_{0}$. For a 3D photonic crystal, in the isotropic model, the DOS exhibits a singularity at the gap edge as proportional to $\left(\omega-\omega_{c}\right)^{-1 / 2}$. Then the Weisskopf-Wigner is not applicable any more. However, according to numerical simulation for 3D photonic crystals, the isotropic model and modified anisotropic model is too much simplified, and gives rise to doubtful QED properties.

To show this, we first consider a photonic crystal with diamond lattice of dielectric spheres in air. This structure is the first one predicted to possess a full band gap in the 
whole Brillouin zone [14]. We have calculated the DOS and LDOS for such structures. The DOS for a diamond lattice of dielectric spheres in air with a relative permittivity of $\epsilon=12.96$ and a filling fraction of $f=0.34$ is displayed in Fig. 1(a). The result is obtained by an expansion of 721 plane waves and by discretizing the first Brillouin zone by over 13000 cells. The curve is plotted with a step of $0.002(2 \pi c / a)$, where $a$ is the lattice constant, $c$ is the light speed in vacuum. The convergence accuracy is better than $0.5 \%$. There appears a large full band gap at the frequency range of $0.440-0.511(2 \pi c / a)$ with a normalized gap size of $14.9 \%$. It is shown that the bottom and top gap edges lie at high symmetry points in the Brillouin zone as $W$ and $L$ points, and their equivalent points, respectively.

The DOS varies slowly near both the bottom and top gap edges. The variation can be approximately fitted by a parabolic curve $\left[\rho(\omega) \propto\left(\omega-\omega_{c}\right)^{2}\right]$ near the band edge [in a frequency range of about $0.04(2 \pi c / a)$ ]. This is in vast contrast to that predicted by the isotropic model [see the inset of Fig. 1(a)]: No singular behavior occurs. In fact, as the gap edge lies at only a limited point in the Brillouin zone (8 equivalent $L$ points and $24 \mathrm{~W}$ points), this nonsingular character of DOS is natural from the physical viewpoint. In addition, the DOS varies much in a wide frequency range. In the long-wavelength regime, it is parabolic, while at higher frequency, there appear some modest peaks. Obviously the approximation of extending the

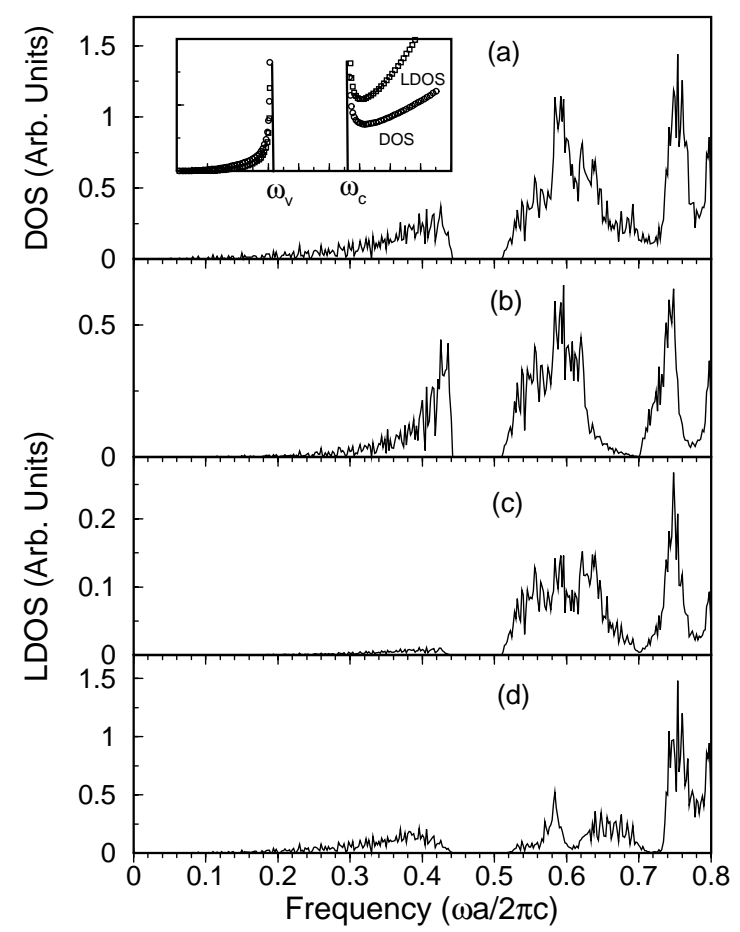

FIG. 1. Plots of (a) DOS and LDOS at sites of (b) $(0,0,0)$, (c) $(0.25 a, 0.25 a, 0.25 a)$, and (d) $(0.5 a, 0,0)$, for a diamond lattice of dielectric spheres in air with $\epsilon=12.96$ and $f=0.34$. Inset in (a): Schematic plots of DOS and LDOS predicted by the isotropic model. dispersion relation near the gap edge to a large frequency regime is thoroughly bad.

According to Eq. (8), the relevant physical function directly correlated with atomic QED is the LDOS, rather than the DOS. Corresponding to Fig. 1(a), we have calculated the LDOS for the atom lying at different sites inside the unit cell of crystal. The convergence is slower than the DOS calculations, yet still satisfactory. The results for LDOS at $\mathbf{r}=(0,0,0),(0.25 a, 0.25 a, 0.25 a)$, and $(0.5 a, 0,0)$ are plotted in Figs. 1(b), 1(c), and 1(d), respectively. In the calculations, we have assumed that the atomic dipole moment is randomly oriented in space. Clearly all the LDOS curves do not follow the variation of the DOS curve; furthermore, they differ remarkably with each other (note the $y$-axis unit of the three panels is the same). All these characters demonstrate that the field-atom coupling coefficient strongly depends on the Bloch wave vector, its magnitude, and orientation. This difference is not expected from the isotropic model, where the vectorial character of the EM wave is completely omitted.

The LDOS variation is also in vast contradiction to inverse square root behavior in the isotropic model [see the inset of Fig. 1(a)]. One can see from Figs. 1(b), 1(c), and 1(d) that the LDOS vanishes in the band gap, a natural result as no states exist, while at frequency near the gap edge, the LDOS increases from zero, basically in a parabolic way. Furthermore, the growth speed differs much at different sites. It is fastest at the site of $\mathbf{r}=(0,0,0)$, while slowest at $\mathbf{r}=(0.25 a, 0.25 a, 0.25 a)$. Irrespective of large difference in the LDOS variation for different sites, a direct extension of LDOS behavior near the gap edge to the whole frequency space is a very poor approximation at each site.

The above analyses based on realistic numerical calculation therefore demonstrate that the approximations made in previous works based on a greatly simplified isotropic model are incorrect even in quality; thus, the predicted QED properties for a 3D photonic crystal are quite problematic. For atom spontaneous emission discussed in this paper concerning the solution of Eq. (8), the WeisskopfWigner approximate theory is still applicable, as the DOS and more accurately the LDOS vary slowly near the gap edge, and far from rapidly in the large frequency range. Then, the solution of Eq. (8) also yields Eq. (9), except that the LDOS for vacuum is now displaced by that in photonic crystals. Therefore, similar to in vacuum, the spontaneous emission of atom in a 3D photonic crystal also exhibits a simple exponentially decaying feature, with a rate of $\Gamma=2 \pi \rho\left(\omega_{0}, \mathbf{r}\right)$. In the band gap $\Gamma=0$, the excited state will persist to any long time, unless other perturbation occurs. This character of spontaneous emission is significantly different from the oscillatory behavior predicted by the isotropic model [8], where the atom-field interaction is much overestimated near the gap edge and therefore qualitative discrepancy is produced.

We now turn to a practical 3D photonic crystal that can be overcome in optical regime: inverse-opal photonic 


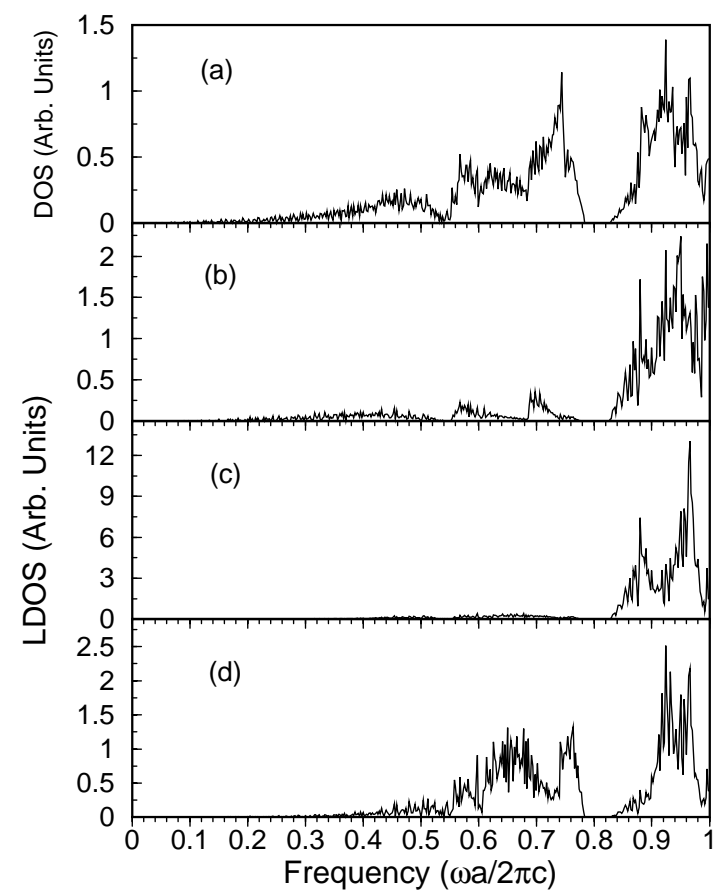

FIG. 2. Plots of (a) DOS and LDOS at sites of (b) $(0,0,0)$, (c) $(0.5 a, 0,0)$, and (d) $(0.36 a, 0.36 a, 0.36 a)$, for an inverse-opal photonic crystal with $\epsilon=12.96$ and $f=0.78$.

crystals [6]. This structure is composed of a face-centered cubic lattice of over-close-packed air sphere in a dielectric background with a high refractive index and opens a full band gap in high frequency photonic bands. We have calculated the DOS and LDOS for such a crystal to investigate the atom spontaneous emission. The results for $\epsilon=12.96$ and $f=0.78$ are displayed in Fig. 2, for (a) the DOS, and LDOS at sites of (b) $\mathbf{r}=(0,0,0)$, (c) $\mathbf{r}=(0.5 a, 0,0)$, and (d) $\mathbf{r}=(0.36 a, 0.36 a, 0.36 a)$, respectively. In the calculations, 729 plane waves and 13000 discretization cells are used, and the dipole moment is assumed to randomly orient. Similar to Fig. 1, the DOS and LDOS curves differ much from each other, and no singular behavior is present. This is in good agreement with previous calculations $[16,17]$. Near the gap edge, the curves vary slowly, which means that the spontaneous emission in such a photonic crystal also exhibits a simple exponentially decaying character with a rate of $\Gamma=2 \pi \rho\left(\omega_{0}, \mathbf{r}\right)$. Further calculations show that the LDOS at other sites all appears to have similar characteristics. It should be noted that current theoretical analyses are in accord with previous observations on molecular spontaneous emission in a 3D photonic crystal, where the dynamics can well be fitted by one or several exponentially decaying curves [11-13].
In summary, we have studied atom spontaneous emission from a 3D photonic crystal in a full vectorial framework by quantizing the Bloch states with use of a plane-wave expansion method. Numerical calculations show that the photon DOS and LDOS for 3D photonic crystals with a full band gap vary slowly near the band gap edge. This is in significant contrast to the singular character predicted by the previous isotropic model. Then, the spontaneous emission can be solved by conventional Weisskopf-Wigner approximate theory. This yields a pure exponentially decaying behavior of spontaneous emission with a rate proportional to the LDOS in the crystals, also appreciably different from the oscillatory feature predicted before. Such a full vectorial treatment should open a way to solving quantitatively QED problems for atoms in photonic crystals.

This work was supported by Hong Kong RGC Grant No. HKUST $6112 / 98 \mathrm{P}$ as well as the National Natural Science Foundation of China.

[1] J. D. Joannopoulos, R. D. Meade, and J. N. Winn, Photonic Crystals (Princeton University Press, Princeton, 1995).

[2] E. Yablonovitch, Phys. Rev. Lett. 58, 2059 (1987).

[3] S. John, Phys. Rev. Lett. 58, 2486 (1987).

[4] S. Y. Lin et al., Nature (London) 394, 251 (1998).

[5] S. Noda et al., Appl. Phys. Lett. 75, 905 (1999).

[6] J.E. G. J. Wijnhoven and W.L. Vos, Science 281, 802 (1998).

[7] S. John and J. Wang, Phys. Rev. Lett. 64, 2418 (1990); Phys. Rev. B 43, 12772 (1991).

[8] S. John and T. Quang, Phys. Rev. A 50, 1764 (1994); T. Quang and S. John, Phys. Rev. A 56, 4273 (1997), and references therein.

[9] S. Y. Zhu, H. Chen, and H. Huang, Phys. Rev. Lett. 79, 205 (1997).

[10] S. Y. Zhu et al., Phys. Rev. Lett. 84, 2136 (2000).

[11] J. Martorell and N. M. Lawandy, Phys. Rev. Lett. 65, 1877 (1990).

[12] E. P. Petrov et al., Phys. Rev. Lett. 81, 77 (1998).

[13] M. Megens et al., Phys. Rev. A 59, 4727 (1999).

[14] K. M. Ho, C. T. Chan, and C. M. Soukoulis, Phys. Rev. Lett. 65, 3152 (1990); Z. Zhang and S. Satpathy, ibid. 65, 2650 (1990); K. M. Leung and Y. F. Liu, ibid. 65, 2646 (1990).

[15] Z. Y. Li, J. Wang, and B. Y. Gu, Phys. Rev. B. 58, 3721 (1998).

[16] K. Busch and S. John, Phys. Rev. E 58, 3896 (1998).

[17] T. Suzuki and P. K. L. Yu, J. Opt. Soc. Am. B 12, 570 (1995).

[18] J. Hanna, M. Watanable, and E. Kato, J. Phys. Condens. Matter 2, 7445 (1990). 